\title{
THE ASYMPTOTIC JOINT DISTRIBUTION OF WINDINGS OF PLANAR BROWNIAN MOTION
}

\author{
BY J. W. PITMAN ${ }^{1}$ AND M. YOR ${ }^{1}$
}

Let $Z=(Z(t), t \geq 0)$ be a complex valued Brownian motion starting at $z_{0}$, and consider the real valued winding processes $\theta_{1}, \theta_{2}, \ldots, \theta_{n}$, where $\theta_{j}(t)$ is the continuous total angle wound by $Z$ about $z_{j}$ up to time $t$, and $z_{0}, z_{1}, \ldots, z_{n}$ are $n+1$ distinct points in the complex plane $\mathbf{C}$. Let $C_{1}, \ldots, C_{n}, B, B^{\infty}$ be $n+2$ mutually independent one-dimensional processes, where the first $n$ are standard Cauchy processes and the last 2 are standard Brownian motions.

Let

$$
\begin{aligned}
T & =\inf \left\{t:\left|B_{t}\right|=1\right\}, \\
U & =\text { local time at } 0 \text { of } B \text { up to time } T, \\
W_{j} & =C_{j}(U)+B^{\infty}(T), \quad j=1, \ldots, n .
\end{aligned}
$$

Theorem 1. As $t \rightarrow \infty$, the $n$-tuple $\left(2 \theta_{j}(t) / \log t, 1 \leq j \leq n\right)$ converges in distribution to $\left(W_{j}, 1 \leq j \leq n\right)$.

The joint Fourier transform of the distribution of $\left(W_{1}, \ldots, W_{n}\right)$ is readily computed to be

$$
E \exp \left(i \sum_{j} \lambda_{j} W_{j}\right)=\left[\cosh \sigma+\left(\sum_{j}\left|\lambda_{j}\right|\right) \frac{\sinh \sigma}{\sigma}\right]^{-1}
$$

where $\sigma=\sum_{j} \lambda_{j}$. Thus for each $j$ the marginal distribution of $W_{j}$ is Cauchy with parameter 1 , and we recover from Theorem 1 the result of Spitzer $[\mathbf{S}]$ for windings about a single point. Theorem 1 is an immediate corollary of Theorem 2 below, which is an extension of Theorem 7 of Messulam and Yor [MY]. To state this result, fix radii $0<r_{j}<\infty, j=1, \ldots, n$, let $D_{j}^{0}$ be the open disc centered at $z_{j}$ with radius $r_{j}$, and let $D_{j}^{\infty}$ be the complement of $D_{j}^{0}$ in C. For ${ }^{*}=0$ or $\infty$, define

$$
\theta_{j}^{*}(t)=\int_{0}^{t} d \theta_{j}(s) 1\left(Z_{s} \in D_{j}^{*}\right)
$$

TheOREM 2. Ast $t \rightarrow \infty$, the $n$-tuple of pairs $\left(2 \theta_{j}^{0}(t) / \log t, 2 \theta_{j}^{\infty}(t) / \log t ; 1 \leq\right.$ $j \leq n)$ converges in distribution to $\left(C_{j}(U), B^{\infty}(T) ; 1 \leq j \leq n\right)$.

Received by the editors July 26, 1983.

1980 Mathematics Subject Classification. Primary 60 J65.

${ }^{1}$ Research supported by NSF Grant MCS-82-02552.

(C) 1984 American Mathematical Society $0273-0979 / 84 \$ 1.00+\$ .25$ per page 
A key step in the proof of Theorem 2 is the use of the pinching method of D. Williams $[\mathbf{W}]$ to show that the asymptotic behavior of

$$
2 \theta_{j}^{*}(t) / \log t \text { as } t \rightarrow \infty
$$

is identical to that of

$$
\theta_{j}^{*}\left(T_{R}\right) / \log R \text { as } R \rightarrow \infty,
$$

where $T_{R}=\inf \left\{t:\left|Z_{t}\right|=R\right\}$.

The identical limiting behavior of (2) and (3) should be contrasted with the different limiting behavior described by Lyons and McKean [LM] for

$$
\theta_{j}^{*}\left(\tau_{u}\right) / u \text { as } u \rightarrow \infty,
$$

where $\tau_{u}$ is the inverse of an additive functional $A_{t}$ with finite representing measure $\alpha$. Let $C^{\infty}\left(\frac{1}{2}\right)$ be a Cauchy random variable with parameter $\frac{1}{2}$ which is independent of the independent and identically distributed random variables $C_{1}\left(\frac{1}{2}\right), \ldots, C_{n}\left(\frac{1}{2}\right)$. The following theorem is the extension to windings about $n$ points of the result given by Lyons and McKean $[\mathbf{L M}]$ for the asymptotic behavior of (4) in the case $n=2$.

TheOREM 3. As $u \rightarrow \infty$, the $n$-tuple of pairs $\left((\alpha(\mathbf{C}) / 4 \pi u)\left[\theta_{j}^{0}\left(\tau_{u}\right), \theta_{j}^{\infty}\left(\tau_{u}\right)\right]\right.$; $1 \leq j \leq n)$ converges in distribution to $\left(\left[C_{j}\left(\frac{1}{2}\right), C^{\infty}\left(\frac{1}{2}\right)\right] ; 1 \leq j \leq n\right)$.

In particular, comparison of the Fourier transform (1) with the corresponding transform for $\left(C_{j}\left(\frac{1}{2}\right)+C^{\infty}\left(\frac{1}{2}\right), 1 \leq j \leq n\right)$ reveals the striking result that while for each $j$ the marginal limit distribution for $2 \theta_{j}(t) / \log t$ is the same Cauchy distribution as for $\alpha(\mathbf{C}) \theta_{j}\left(\tau_{u}\right) / 4 \pi u$, the joint limiting distributions of these quantities are different.

An important ingredient in our proof of these results is the independence of various radial and angular parts of the Brownian motion as described in Theorem 4 below. We derive this theorem using Tanaka's formula and the theorem of Knight [K] representing orthogonal continuous martingales in terms of independent Brownian motions.

For each $j=1, \ldots, n,{ }^{*}=0$ or $\infty$, introduce the additive functional

$$
U_{j}^{*}(t)=\int_{0}^{t} 1\left(Z_{s} \in D_{j}^{*}\right)\left|Z_{s}-z_{j}\right|^{-2} d s
$$

and the clock

$$
t_{j}^{*}(u)=\inf \left\{t: U_{j}^{*}(t)=u\right\},
$$

and define time changed processes

$$
\beta_{j}^{*}(u)=\theta_{j}^{*}\left(t_{j}^{*}(u)\right), \quad \rho_{j}^{*}(u)=\log \left|Z\left(t_{j}^{*}(u)\right)-z_{j}\right| .
$$

TheOREM 4. (i) For each $j=1, \ldots, n,{ }^{*}=0$ or $\infty, \beta_{j}^{*}$ is a Brownian motion, $\rho_{j}^{*}$ is a Brownian motion with reflection at $\log r_{j}$, and these two processes are independent.

(ii) If the domains $D_{1}^{0}, \ldots, D_{n-1}^{0}, D_{n}^{\infty}$ are disjoint, then the $2 n$ processes $\beta_{1}^{0}, \rho_{1}^{0}, \ldots, \beta_{n-1}^{0}, \rho_{n-1}^{0}, \beta_{n}^{\infty}, \rho_{n}^{\infty}$ are mutually independent. 
For the proof of Theorems 2 and 3, it is an important observation that the origins $z_{j}$ for the angles and the radii $r_{j}$ separating $\theta_{j}^{\infty}$ and $\theta_{j}^{0}$ play no rôle in the limit. We prove this using estimates relating martingales to their increasing processes (see, e.g., Burkholder $[\mathbf{B}]$ ). This allows reduction to the case when the domains $D_{1}^{0}, \ldots, D_{n-1}^{0}, D_{n}^{\infty}$ are disjoint. In this case we analyse the angles indexed by local times on the $n$ circles, making use of the independence results of Theorem 4 and the ratio ergodic theorem for additive functionals (see, e.g., Ito and McKean [IM, §17]).

The Cauchy processes arise from the fact that if $\tau_{j u}^{*}$ denotes the inverse local time process on the boundary circle of $D_{j}^{*}$, then $\left(\theta_{j}^{*}\left(\tau_{j u}\right), u \geq 0\right)$ is a Cauchy process.

ACKnOWLedgement. We thank David Aldous for developing an elaborate coupling argument which completed our first proof of the asymptotic distribution of $\theta_{1}^{0}(t)$ and $\theta_{2}^{0}(t)$.

\section{REFERENCES}

[B] D. Burkholder, Distribution function inequalities for martingales, Ann. Probab. 1 (1973), 19-42.

[IM] K. Ito and H. P. McKean, Diffusion processes and their sample paths, Springer-Verlag, 1965.

[K] F. B. Knight, A reduction of continuous square-integrable martingales to Brownian motion, Lecture Notes in Math., vol. 190, Springer-Verlag, 1971.

[LM] T. Lyons and H. P. McKean, Winding of the plane Brownian motion (to appear).

[MY] P. Messulam and M. Yor, On D. Williams' "Pinching method" and some applications, J. London Math. Soc. (2) 26 (1982), 348-364.

[S] F. Spitzer, Some theorems concerning 2-dimensional Brownian motion, Trans. Amer. Math. Soc. 87 (1958), 187-197.

[W] D. Williams, A simple geometric proof of Spitzer's winding number formula for 2-dimensional Brownian motion, University College, Swansea, 1974, unpublished manuscript.

Department of Statistics, University of California, Berkeley, California 94720

Current address (M. Yor): Laboratoire de Calcul des Probabilités \#4, Place Jussieu, Tour 56, 75230 Paris Cedex 05, France 
